OPEN ACCESS

Edited by:

Alberto Enrique Paniz Mondolfi, Icahn School of Medicine at Mount Sinai, United States

Reviewed by: John Mina,

Teesside University, United Kingdom Isabel Mauricio,

New University of Lisbon, Portugal

*Correspondence:

Paola Andrea Barroso paolabarroso@conicet.gov.ar; barrosopaola75@gmail.com

Specialty section: This article was submitted to Parasite and Host,

a section of the journal Frontiers in Cellular and Infection Microbiology

Received: 11 February 2021 Accepted: 17 May 2021 Published: 04 June 2021

Citation:

García-Bustos MF, Moya Álvarez A, Pérez Brandan C, Parodi C, Sosa AM, Buttazzoni Zuñiga VC, Pastrana OM, Manghera $P$, Peñalva PA, Marco JD and Barroso PA (2021) Development of a Fluorescent Assay to Search New Drugs Using Stable tdTomato-

Leishmania, and the Selection of Galangin as a Candidate With Anti-Leishmanial Activity. Front. Cell. Infect. Microbiol. 11:666746. doi: 10.3389/fcimb.2021.666746

\section{Development of a Fluorescent Assay to Search New Drugs Using Stable tdTomato-Leishmania, and the Selection of Galangin as a Candidate With Anti-Leishmanial Activity}

\author{
María Fernanda García-Bustos ${ }^{1,2,3}$, Agustín Moya Álvarez², Cecilia Pérez Brandan², \\ Cecilia Parodi ${ }^{2}$, Andrea Mabel Sosa ${ }^{2}$, Valeria Carolina Buttazzoni Zuñiga ${ }^{1}$, \\ Oscar Marcelo Pastrana ${ }^{1}$, Paula Manghera ${ }^{1}$, Pablo Alejandro Peñalva ${ }^{1}$, Jorge Diego Marco ${ }^{2}$ \\ and Paola Andrea Barroso ${ }^{2 *}$

\begin{abstract}
1 Escuela Universitaria en Ciencias de la Salud y Facultad de Ciencias Agrarias y Veterinarias, Universidad Católica de Salta, Salta, Argentina, ${ }^{2}$ Instituto de Patología Experimental, Consejo Nacional de Investigaciones Científicas y Técnicas (CONICET) - Universidad Nacional de Salta, Salta, Argentina, ${ }^{3}$ Facultad de Ciencias de la Salud, Universidad Nacional de Salta, Salta, Argentina
\end{abstract}

Antimonials continue to be considered the first-line treatment for leishmaniases, but its use entails a wide range of side effects and serious reactions. The search of new drugs requires the development of methods more sensitive and faster than the conventional ones. We developed and validated a fluorescence assay based in the expression of tdTomato protein by Leishmania, and we applied this method to evaluate the activity in vitro of flavonoids and reference drugs. The pIR1SAT/tdTomato was constructed and integrated into the genome of Leishmania (Leishmania) amazonensis. Parasites were selected with nourseothricin (NTC). The relation of $L$. amaz/tc3 fluorescence and the number of parasites was determined; then the growth in vitro and infectivity in BALB/C mice was characterized. To validate the fluorescence assay, the efficacy of miltefosine and meglumine antimoniate was compared with the conventional methods. After that, the method was used to assess in vitro the activity of flavonoids; and the mechanism of action of the most active compound was evaluated by transmission electron microscopy and ELISA. A linear correlation was observed between the emission of fluorescence of $L$. amaz/tc3 and the number of parasites $\left(r^{2}=0.98\right)$, and the fluorescence was stable in the absence of NTC. No differences were observed in terms of infectivity between $L$. amaz/tc3 and wild strain. The efficacy of miltefosine and meglumine antimoniate determined by the fluorescence assay and the microscopic test showed no differences, however, in vivo the fluorescence assay was more sensitive than limiting dilution assay. Screening assay revealed that the flavonoid galangin (GAL) was the most active compound with $\mathrm{IC}_{50}$ values of $53.09 \mu \mathrm{M}$ and $20.59 \mu \mathrm{M}$ in promastigotes and intracellular amastigotes, respectively. Furthermore, GAL induced mitochondrial swelling, lipid inclusion bodies and vacuolization in promastigotes; and up-modulated the production of IL-12 p70 in infected 
macrophages. The fluorescence assay is a useful tool to assess the anti-leishmanial activity of new compounds. However, the assay has some limitations in the macrophageamastigote model that might be related with an interfere of flavanol aglycones with the fluorescence readout of tdTomato. Finally, GAL is a promising candidate for the development of new treatment against the leishmaniasis.

Keywords: tdTomato, L. (L.) amazonensis, flavonoids, galangin, fluorescence

\section{INTRODUCTION}

The leishmaniases are a group of diseases caused by parasites of the genus Leishmania, which are transmitted to humans through the bite of infected female phlebotomine sandflies. These diseases in all their clinical expressions are endemic in 98 countries (Alvar et al., 2012), affecting mainly low income populations of the tropical and subtropical belt worldwide. According to the WHO report (2016), over one billion people live in endemic areas at risk of infection (World Health Organization, 2016), and an estimated $900.000-1.6$ million new cases and 20.000 to 40.000 deaths occur annually (Alvar et al., 2012).

Depending on the infecting Leishmania species and the immune response of the host, leishmaniases can exhibit a disease spectrum that includes visceral leishmaniasis or "kalaazar", affecting mainly the mononuclear phagocytic system; as well as cutaneous (cutaneous leishmaniasis, CL) and mucosal forms (mucosal leishmaniasis, ML), which are characterized by involvement of skin and mucous membranes, respectively (Mcgwire and Satoskar, 2014).

The development of new therapeutic approaches for leishmaniasis treatment requires as well new high throughput screening methodologies for searching compounds with antileishmanial activity. Reporter gene (RG) technology has become one of the most promissory and widely used tool for drug screening in several models since it offers live imaging, high sensibility, specificity and flexibility (Pulido et al., 2012). These genes typically encode a gene product that has a readily measurable phenotype and is easily distinguishable over endogenous cellular background (Dube et al., 2009). Depending on the application, an ideal RG should be: (i) absent from the host; (ii) inert (should not affect the physiology of the parasite cell); and (iii) should represent a simple, sensitive, and inexpensive assay for quantification of reporter expression (Dube et al., 2009). The choice of a RG depends on the cell line used, the nature of the experiment, and the adaptability of the assay to the appropriate detection method (New et al., 2003).

Fluorescent RGs have been applied in the developing of new methods less laborious and more sensitive than the classical microscopy method for drug screening. The family of green fluorescent protein (GFP and multimeric gfp) was the most studied, but the main disadvantage of these RGs was the low level of fluorescence of transgenic parasites (Ha et al., 1996). Moreover, a background noise was observed, when $L$. (L.) donovani expressing the episomal GFP was used for the screening in intracellular amastigotes and promastigotes generating a problem to automatize the technique (Dube et al., 2005). Another approach was to use the enhanced GFP (egfp) trying to improve the fluorescent signals. The transfected parasites showed stable expression of the fluorescent protein and the assay provided a more accurate approach in drug sensitivity profile, but it was not automated as a high-throughput (Bolhassani et al., 2011). Then, GFP integrated into the Leishmania genome was evaluated, the expression was very stable and homogeneous reducing the noise generated by similar episomal genetic manipulation (Pulido et al., 2012). This allows the development of automatized methodologies with the least background. Another RG, is tandem dimer Tomato (tdTomato), a variant of DsRed, derived from the coral Discosoma $\mathrm{sp}$, which has demonstrated photostability and a high brightness (283\% of eGFP) (Shaner et al., 2004; Morris et al., 2010). TdTomato has been used successfully in transgenic mouse models, fusion protein applications, and as a promoter-reporter (Morris et al., 2010). It also demonstrated to be useful in the generation of Trypanosoma cruzi parasite lines that express this fluorescent protein and the use of these lines to establish accurate and simple in vitro as well as in vivo systems to screen and test anti-T. cruzi compounds (Canavaci et al., 2010).

On the other hand, chemotherapy remains the major control strategy for leishmaniases with meglumine antimoniate (Ma) enduring as the first-line treatment (Pan American Health Organization, 2013). However, its use in the clinical setting exhibits several limitations, given that they require an aggressive administration schedule (one or two intramuscular daily injections for 21 to 35 days), and often exhibit a wide range of local and systemic side effects. On the other hand, Pan American Health Organization (PAHO) recognizes that currently none of the drugs available for the treatment of leishmaniasis in the Americas completely eradicates the infection (PAHO, 2013). Given the above-mentioned reasons, the development of new, less toxic and more cost-effective drugs with greater efficacy as well as more accessible alternative therapeutic strategies that could become available for lowincome populations to treat the disease has become a necessity (PAHO, 2013; Oryan, 2015).

In recent years, there has been growing interest in alternative natural products and plant compounds for the treatment of leishmaniases (Chouhan et al., 2014). The anti-leishmanial activity of some plant extracts has been attributed to flavonoids (Wong et al., 2014). These are a group of polyphenolic compounds that naturally present in fruits and vegetables and are known as antioxidants and anticancer with a significant protective effect against membrane damage (Sifaoui et al., 2014). 
Recently, we reported the activity of several catechins from Camellia sinensis against L. (L.) amazonensis, being EGCG the most active one (Sosa et al., 2020). This group of compound can form complexes with the parasite cell wall to influence processes requiring cell linking, and hence inhibit the parasite growth (Ogeto et al., 2013). In a research work carried out by Manjolin et al. (2013), it has been shown that dietary flavonoids with low cytotoxicity characteristics such as fisetin, quercetin, luteolin and 7,8-hydroxyflavone can inhibit arginase enzyme from L. (L.) amazonensis. Arginase plays a central role in the biosynthesis of polyamine which is very important and essential for protecting the parasite against oxidative stress and ROS produced by the host's defense system (Oryan, 2015). Therefore, natural compounds as flavonoids are an interesting group to search potential candidate with anti-leishmanial activity.

For all the aforementioned, the aims of this work were to develop and validate a fluorescence assay based in the expression of tdTomato protein by Leishmania (L.) amazonensis, and to apply this method to evaluate in vitro the activity of flavonoids and reference drugs. In addition, we studied the mechanism of action of one active compound selected though the in vitro screening assay.

\section{MATERIALS AND METHODS}

\section{Compounds}

Flavonoids: Flavonol: Galangin (GAL) - $\geq 95 \%$ (HPLC) (SigmaAldrich 282200), fisetin (FI) - P90\% (HPLC) (PhytoLab PHL82542), rutin (RU) - >95.0\% (HPLC) (PhytoLab PHL89270), morin (MO) $-\geq 95.0 \%$ (HPLC) (PhytoLab PHL82601). Flavanol: (-)-epigallocatechin gallate (EGCG)- $>98 \%$ (HPLC) was kindly supplied by Mitsui Norin (Shizuoka, Japan).

Reference drugs: Pentamidine isethionate (PE) (SigmaAldrich P0547) and Miltefosine (MI) - $\geq 98.0 \%$ (perchloric acid titration) (Sigma-Aldrich M5571) were purchased from SigmaAldrich. Meglumine antimoniate $(\mathrm{Ma})$ (Glucantime ${ }^{\circledR}$, AventisSanofi, São Paulo, Brazil) was kindly supplied by Ministerio de Salud de la Nación (Buenos Aires, Argentina).

\section{Parasites}

Parasites of L. (L.) amazonensis (MHOM/BR/73/M2269) were isolated from an infected mouse. First, the isolated parasites were cultured in Difco agar containing 20\% defibrinated rabbit blood (USMARU medium) plus sterile proline balanced salts solution (PBSS) with $100 \mathrm{U} / \mathrm{mL}$ penicillin and $50 \mu \mathrm{g} / \mathrm{mL}$ streptomycin (P$\mathrm{S})$ at $23^{\circ} \mathrm{C}$. After four days, the parasites were passaged to RPMI1640 medium (Gibco, Grand Island, NY, USA) supplemented with $10 \%(\mathrm{v} / \mathrm{v})$ heat-inactivated fetal bovine serum (FBS) and P-S.

\section{PIR1SAT/tdTomato Plasmid Construction}

The tandem dimeric tomato red fluorescent reporter gene (tdTomato, $1464 \mathrm{bp}$ ) was obtained by restriction enzyme digestion from the pTREX-tdTomato plasmid constructed for Trypanosoma cruzi (Canavaci et al., 2010). The fragment was then gel purified and cloned into the expression site of pIR1SAT plasmid (Xba and Sal1). Correct insertion of the tdTomato gene was corroborated by digestion with selected restriction enzymes. For transfection, pIR1SAT-tdTomato plasmid was linearized with SwaI enzyme, gel purified and quantified. This plasmid integrates into the Leishmania genome by replacing one copy of the SSU rRNA gene which is transcribed by poll (Buckner and Wilson, 2005). pTREX-tdTomato and pIR1SAT plasmids were kindly provided by Dr. R.L. Tarleton (University of Georgia, USA) and Dr. S. Beverley (Washington University, USA), respectively. Additional information about tdTomato and its sequence can be found at the following link https://www. fpbase.org/protein/tdtomato/.

\section{Transfection of Leishmania}

Mid-log promastigotes of $L$. (L.) amazonensis were collected by centrifugation and washed once with phosphate buffer saline (PBS; pH 7.4) plus P-S. Then, parasites were resuspended in cold OPTI-MEM reduced serum medium (Gibco) at $1 \times 10^{8} / \mathrm{ml}$ and the pIR1SAT-tdTomato $(10 \mu \mathrm{g} / \mathrm{ml})$ was added. The parasites and the plasmid were placed in a $2 \mathrm{~mm}$ gap electroporation cuvette (BTX Inc., San Diego, CA). The cuvette was chilled on ice for $10 \mathrm{~min}$, then parasites were electroporated using an Electro Cell Manipulator 630 (BTX Inc.) set at $450 \mathrm{~V}, 0.025 \Omega \mathrm{l}$, and $500 \mu \mathrm{F}$. After electroporation, the cuvette was placed on ice for $10 \mathrm{~min}$, then the parasites were transferred to USMARU medium for $24 \mathrm{~h}$. One-day post-electroporation, nourseothricin (NTC) (50 $\mu \mathrm{g} / \mathrm{ml}$ ) was added to select parasites expressing the streptothricin acetyltransferase (SAT) gene. Resistant parasites were cloned in a Petri dish in USMARU medium plus RPMI-1640 with 10\% SFB and NTC $(50 \mu \mathrm{g} / \mathrm{ml})$. A diagnostic PCR was carried out in 6 clones (C2, C3, C5, C11, C12 and C21) to confirm the insertion of tdTomato gene into the Leishmania genome. The sequence of the primers used is detailed in Supplementary Material.

\section{Detection of Fluorescence Signal in Transfected Parasites and Its Correlation With the Number of Parasites}

The fluorescence emission was measured in C2, C3, C5, C11, C12 and C21. Briefly, promastigotes in the log phase $\left(1 \times 10^{6} / 0.1 \mathrm{ml}\right)$ of each clone and promastigotes of the wild-type strain (L. amaz) wt) were plated in a 96-well black culture plate with clear bottom (Nunc, USA) and incubated at $23^{\circ} \mathrm{C}$. After $24 \mathrm{~h}$ of incubation, the fluorescence was measured in a fluorimeter plate reader Infinite F200 (Tecan, Männedorf, Switzerland) with the optics positioned under the bottom of the plate and the filters 535/25 for excitation and 595/35 for emission. A clone (L. amaz/tc3) was selected for subsequent in vitro and in vivo experiments.

To determine the correlation between the fluorescence signal and the number of parasites, L. amaz/tc3 promastigote in the mid-log phase $\left(3 \times 10^{7}, 1 \times 10^{7}, 3 \times 10^{6}, 1 \times 10^{6}, 3 \times 10^{5}, 1.2 \times 10^{5}\right.$, $4 \times 10^{4}$ cells/well) and L. amaz/tc3 amastigotes $\left(3 \times 10^{6}, 1 \times 10^{6}\right.$, $3 \times 10^{5}, 1 \times 10^{5}, 3 \times 10^{4}, 1.2 \times 10^{4}, 4 \times 10^{3}$ cells/well) were plated in $0.1 \mathrm{ml}$ in a 96 -well black culture plate with clear bottom (Nunc, USA). In the case of amastigotes, the parasites were isolated from a macrophage culture as was described previously (Chang, 1980). 
Un-transfected parasites ( L. amaz/wt) were the negative control. The fluorescence was measured in a fluorimeter plate reader (Tecan, Männedorf, Switzerland).

\section{In Vitro Assessment of Transfected Parasites Growth}

In order to evaluate if the growth of $L$. amaz/tc3 was modified by the transfection with the RG, promastigotes $\left(5 \times 10^{5} \mathrm{p} / \mathrm{ml}\right)$ were plated in triplicate in RPMI-1640 medium in a 96-well culture plate and the positive control was $L$. amaz/wt. At the $1^{\text {st }}, 3^{\text {rd }}$ and $7^{\text {th }}$ day of incubation, the density (parasites $/ \mathrm{ml}$ ) was determined using a Neubauer chamber in an optic microscope (40x) (Leitz Wetzlar, Germany).

Then, the growth of intracellular amastigotes of L. amaz/tc3 was evaluated. Raw 264.7 cells ( $5 \times 10^{4} /$ well) were cultured in complete RPMI-1640 and plated in 96-well black plate (Nunc, USA) for $3 \mathrm{~h}$ at $37^{\circ} \mathrm{C}$ in a $5 \% \mathrm{CO}_{2}$ for macrophage adherence. After that, macrophages were infected with promastigotes of $L$. amaz/tc3 at different ratios, 1:5, 1:10 and 1:20 (macrophage: promastigotes), and incubated at $34^{\circ} \mathrm{C}$ in a $5 \% \mathrm{CO}_{2}$ overnight. Infected cultures were washed with pre-warmed PBS to remove free parasites. Macrophages without infection were the negative controls. The fluorescent was measured in a plate reader (Tecan, Männedorf, Switzerland) at $1^{\text {st }}, 2^{\text {nd }}, 3^{\text {rd }}$ and $4^{\text {th }}$ day of culture.

\section{In Vitro, Fluorescent Assay Validation}

Then, we evaluated the efficacy of MI, a reference drug for leishmaniasis by the fluorescence method (L.amaz/tc3) and by the conventional method based in counting the parasite at the optic microscope (L. amaz/wt). Mid-log promastigotes of $L$. amaz/tc3 and L. amaz/wt were harvest and seeded $\left(1 \times 10^{6} \%\right.$ $0.1 \mathrm{ml})$ with different concentrations of $\mathrm{MI}(40$ to $0.6 \mu \mathrm{g} / \mathrm{ml})$ in a 96-well plate at $23^{\circ} \mathrm{C}$. Promastigotes cultured only in medium were the controls. Parasite viability was determined by measuring the fluorescence of $L$. amaz/tc3 in a plate reader (Tecan, Männedorf, Switzerland) and by counting the mobile promastigotes in a Neubauer chamber at the optic microscope (40x) (Leitz Wetzlar, Germany) at $48 \mathrm{~h}$ of incubation with MI. Viability was calculated with the formula: Viability L.amaz/tc3 $_{\text {. }}=$ $\frac{F L t}{F L c} \times 100$ where, FLt is the fluorescence of treated culture, and FLc is the fluorescence of control; Viability L.amaz $/ w t=\frac{N P t}{N P c} \times 100$ where, NPt is the number of promastigotes in the treated culture, and NPc is the number of promastigotes in control culture. Then, the $50 \%$ inhibitory concentration $\left(\mathrm{IC}_{50}\right.$ ) of $\mathrm{MI}$ was estimated.

Furthermore, the efficacy of MI was also determined against intracellular amastigotes of $L$. amaz/tc3 and L. amaz/wt. Raw 264.7 macrophages were plated in 96-well black plate $\left(5 \times 10^{4}\right.$ cells/well) and in eight well Lab-Tek tissue culture slides $(6.6 \mathrm{x}$ $10^{4}$ cells $/ \mathrm{ml}$ ) (Nunc, USA). The cells were infected with promastigotes in stationary phase of L. amaz/tc3 and L. amaz/ wt ( $1: 20$ ratio) at $33^{\circ} \mathrm{C}, 5 \% \mathrm{CO}_{2}$. After incubation, the infected macrophages were washed to remove non-internalized parasites with PBS plus P-S. New RPMI-1640 medium with different concentrations of MI (20 to $0.62 \mu \mathrm{g} / \mathrm{ml}$ ) was added to each well and incubated at $33^{\circ} \mathrm{C}, 5 \% \mathrm{CO}_{2}$ during $48 \mathrm{~h}$. The antileishmanial activity of $\mathrm{MI}$ was determined by measuring the fluorescence of intracellular amastigotes of L. amaz/tc3 in a plate reader (Tecan, Männedorf, Switzerland). The viability was calculated as mentioned above, and the $\mathrm{IC}_{50}$ was also estimated. Simultaneously, the leishmanicidal activity of MI against $L$. amaz/wt was determined by the microscopy test. In this case, the infected macrophages were stained with Diff Quik (Biopur S.R.L., Rosario, Argentina) and the number of intracellular amastigotes was determined in 200-500 macrophages in treated and control cultures per slide under the immersion lens microscope (100x) (Leitz Wetzlar, Germany). The infection index (II) and the \% infectivity (I) were calculated by the following formulas: $I I=I M \times A$, where $\mathrm{IM}=$ infected macrophages (\%), $\mathrm{A}=$ number of amastigotes per infected macrophages; and $I(\%)=\frac{I I T}{I I c} \times 100$, where IIT= infection index in treated cultures, IIC $=$ infection index in control cultures (Ayres et al., 2008). The $\mathrm{IC}_{50}$ of $\mathrm{MI}$ was calculated.

\section{Course of Infection of $L$. amaz/tc3 in Mice}

Mice were bred at the mouse facility of the Faculty of Health Sciences, National University of Salta (Salta, Argentina). Female $\mathrm{BALB} / \mathrm{c}$ mice $(n=5)$ of 6 weeks of age were infected in their hind right footpad with the stationary phase promastigotes $\left(5 \times 10^{6}\right.$ parasites $/ 0.05 \mathrm{ml}$ ) to evaluate the infectivity of $L$. amaz/tc3; animals infected with $L$. amaz/wt were the controls $(n=5)$. The footpad swelling was measured weekly with a digital caliper (150 mm; Digimess).

\section{Efficacy of Ma in Mice Infected With $L$. amaz/tc3}

Female BALB/c mice were infected in their hind right footpad with the stationary phase of promastigotes $\left(5 \times 10^{6}\right.$ parasites/ $0.05 \mathrm{ml}$ ) of $L$. amaz/tc3. Once a week the footpad swelling was measured with the digital caliper. Mice $(n=5)$ were treated with Ma in PBS (120 mg Sb/kg/day) starting at week 9 post-infection; the antimonial was administered by intraperitoneal injection (ip) once a day for 21 days. Control group $(n=5)$ received only PBS (ip). Within a week of finishing the Ma treatment, the parasite load was quantified by three methods, fluorescence (FL), optic microscope (OM) and limiting dilution assay (LD) in treated and control group. The FL method was carried out as follows, the hind right footpad was excised and weighed; then the tissue was homogenized using a glass grinder with $5 \mathrm{ml}$ of complete RPMI1640 medium and the intracellular amastigotes of $L$. amaz/tc3 were released. A sample $(0.1 \mathrm{ml})$ of the homogenate was centrifuged to replace the medium for PBS, then it was plated in duplicates in a 96-well black culture plate with clear bottom (Nunc, USA) and the fluorescence was measured in a plate reader (Tecan, Männedorf, Switzerland). Besides, a calibration curve was used to extrapolate the sample fluorescence to the number of amastigotes. For that, a pool of amastigotes from control samples was used; amastigotes $\left(1 \times 10^{6}\right.$ to $\left.1.5 \times 10^{4}\right)$ were plated in duplicates in a 96-well black culture plate with clear bottom, and the fluorescence was measured in a plate reader (Tecan, Männedorf, Switzerland). The number of amastigotes was adjusted to the weight of the sample. 
Another sample of the tissue homogenate was used to quantify the parasite loads by direct counting in a Neubauer chamber under an optical microscope (40X) (Leitz Wetzlar, Germany), and the concentration of parasites (amastigotes/ml) of each homogenate was determined (Hill et al., 1983). The total number of parasites was adjusted to the weight of each tissue sample.

Finally, a sample of the tissue homogenate was also used to quantify the parasite loads by the LD assay (Lima et al., 1997). After serial dilutions in 96-well plates, the samples were incubated at $23^{\circ} \mathrm{C}$ for 14 days and the number of viable promastigotes was determined from the highest dilution at which the parasite could grow.

\section{Drug Screening Against Promastigotes and Intracellular Amastigotes by the Fluorescence Assay}

After fluorescence assay validation as it is described above, the method was used to evaluate in vitro the flavonoids antileishmanial activity. Mid-log promastigotes of $L$. amaz/tc3 were harvest and seeded with different concentrations of GAL (1,200 to $18.75 \mu \mathrm{M}), \mathrm{MO}, \mathrm{FI}$ and RU (40 to $1.25 \mu \mathrm{M})$, and EGCG (300 to $18.75 \mu \mathrm{M})$. Positive control were promastigotes treated with PE (4 to $0.25 \mu \mathrm{M}$ ), the negative control were promastigotes cultured with medium only, and the blank was the culture medium. The parasites were incubated at $23^{\circ} \mathrm{C}$ for $48 \mathrm{~h}$. The anti-leishmanial activity was determined by measuring the parasite fluorescence in a plate reader (Tecan, Männedorf, Switzerland). The viability was estimated as was described above for MI (fluorescent assay). The $\mathrm{IC}_{50}$ was calculated for each compound.

The flavonoids anti-leishmanial activity was also evaluated against intracellular amastigotes. Macrophages (Raw 264.7) were plated in 96-well black plate $\left(5 \times 10^{4}\right.$ cell/well) and infected with promastigotes in stationary phase of $L$. amaz/tc3 (1:20 ratio) at $33^{\circ} \mathrm{C}, 5 \% \mathrm{CO}_{2}$. After incubation, the infected macrophages were washed to remove non-internalized parasites with PBS plus P-S. New RPMI-1640 medium with different concentrations of GAL (30 to $3.75 \mu \mathrm{M}), \mathrm{MO}$ (300 to $37.5 \mu \mathrm{M})$, FI (65 to $8.12 \mu \mathrm{M})$, RU (300 to $37.5 \mu \mathrm{M}$ ) and EGCG (30 to $7.5 \mu \mathrm{M}$ ) was added to each well and incubated at $33^{\circ} \mathrm{C}, 5 \% \mathrm{CO}_{2}$ for $48 \mathrm{~h}$. Infected cells treated with $\mathrm{Ma}(136.6$ to $34.1 \mu \mathrm{M})$ were the positive controls, and cells incubated only with RPMI-1640 were the negative control. The infected macrophages were incubated during $48 \mathrm{~h}$ at $33^{\circ} \mathrm{C}, 5 \% \mathrm{CO}_{2}$. After treatment, the fluorescence of intracellular amastigotes of $L$. amaz/tc3 was measured in a plate reader (Tecan, Männedorf, Switzerland). The viability was determined as was described for MI (fluorescent assay). The selectivity index (SI) $\left(\mathrm{CC}_{50}\right.$ for macrophages/ $\mathrm{IC}_{50}$ for Leishmania) was calculated. A SI higher than one, was considered more selective for activity against Leishmania, whereas a value lower than one was considered more selective for the activity against the macrophages (Tiuman et al., 2005).

All experiments in promastigotes and intracellular amastigotes were performed in duplicate or triplicate wells for each condition and repeated at least twice.

\section{Drug Toxicity in a Macrophage Cell Line}

The cytotoxicity against a cell line of macrophages (Raw 264.7) of GAL, MO, FI, RU, EGCG, PE, MI and MA was determined by the MTT method. Cells $\left(3.3 \times 10^{5} / \mathrm{ml}\right)$ cells were treated with different concentrations of each compound during $48 \mathrm{~h}$ at $37^{\circ} \mathrm{C}$, $5 \% \mathrm{CO}_{2}$. The controls were incubated with RPMI-1640 medium. Briefly, $50 \mu \mathrm{l}$ of MTT ( $2 \mathrm{mg} / \mathrm{mL}$ ) was added to each well and, after $4 \mathrm{~h}$ of incubation at $37^{\circ} \mathrm{C}, 5 \% \mathrm{CO}_{2}$, the reaction was stopped dissolving the formazan crystals with $100 \mu \mathrm{l} /$ well of dimethyl sulfoxide. The relative amount of formazan produced by viable macrophages was determined using a spectrophotometer at 595 $\mathrm{nm}$. The percentage of viable cells was calculated according to the formula $\frac{\left(O D_{\text {treated culture }}-O D_{\text {blank }}\right)}{\left(O D_{\text {control }}-O D_{\text {blank }}\right)} \times 100$ as was described previously (Adinehbeigi et al., 2017). The cytotoxicity concentration 50\% $\left(\mathrm{CC}_{50}\right)$ was calculated for each compound.

\section{Analysis by Transmission Electron Microscopy}

Ultrastructural alterations in Leishmania induced by flavonoids with anti-leishmanial activity were analyzed by transmission electron microscopy (TEM). Promastigotes of L. amaz/tc3 (1 x $10^{6}$ parasites $/ \mathrm{ml}$ ) were exposed to the $\mathrm{IC}_{50}$ of most active flavonoids selected in the drug screening. The control was a culture of L. amaz/tc3 incubated in complete RPMI-1640 medium. After $48 \mathrm{~h}$ of incubation, samples of L. amaz/tc3 promastigotes from each culture were fixed in modified Karnovsky's solution (formaldehyde [2.66\%], glutaraldehyde [1.66\%]) and PBS 0.1M ( $\mathrm{pH} 7,4)$, and incubated overnight at $4^{\circ} \mathrm{C}$. The fixed samples were post-fixed with a $1 / 1$ solution of $\mathrm{PBS} / 2 \%$ osmium tetroxide (OsO4), overnight. Then the material was dehydrated and included in Spurr (Pelco, USA). Ultrathin sections were cut with an ultramicrotome (MT1, Sorvall, USA), mounted on copper grids, and contrasted with uranyl acetate (PELCO, USA) and lead citrate. The samples were observed with a Zeiss EM109 (Carl Zeiss, Oberkochen, Germany) transmission electron microscope, at CIME (Centro Integral de Microscopía Electrónica, CONICET-UNT-Tucumán, Argentina) and electron micrographs were taken.

\section{Cytokine Production by Activated Macrophages}

To evaluate if the most active flavonoid against Leishmania can active infected macrophages to produce IL-12p70 and IL-10 cytokines, macrophages (Raw 264.7) were infected with promastigotes (ratio 1:20) of $L$. amaz/tc3 as was described previously in "Drug Screening Against Promastigotes and Intracellular Aastigotes by The Fluorescence Assay" section, and cultured in a $5 \% \mathrm{CO}_{2}$ incubator. Non-infected cells $\left(2 \times 10^{5}\right.$ cells/ $\mathrm{mL}$ ) were cultured in RPMI-1640 medium plus 10\% FCS and 1\% antibiotics (P-S) and placed into 24 well plates. Cells were exposed to $5 \mu \mathrm{g} / \mathrm{ml}$ of phytohaemagglutinin (PHA) (Sigma Aldrich, Missouri, USA), $5 \mu \mathrm{g} / \mathrm{ml}$ of concanavalin A (CONA) (Sigma Aldrich, Missouri, USA); and the most active flavonoid selected against Leishmania $(20 \mu \mathrm{M})$ during $48 \mathrm{~h}$. Then, the culture supernatant was recovered and maintained at $-80^{\circ} \mathrm{C}$ until use. The culture supernatant concentrations of IL-12p70 and 
IL-10 (BD Biosciences, San Diego, USA) were determined by ELISA following the specifications of the supplier. Each condition was performed in quadruplicate.

\section{Statistical Analysis}

The GraphPad Prism 5.0 software (GraphPad Software Inc., San Diego, CA, USA) was used to calculate the $\mathrm{IC}_{50}$ and $\mathrm{CC}_{50}$ values by nonlinear regression. Multiple comparisons of the parasite burden between groups were made with a one-way analysis of variance (ANOVA) followed by Tukey test. Comparisons between two groups were performed by Mann-Whitney (nonparametric t-test). The parasite loads obtained by the LD assay were estimated with the ELIDA software (Taswell, 1986). $p<0.05$ was considered significant. For the analyses of culture supernatants by ELISA, continuous variables between two groups were compared with the Mann-Whitney U-test. A nonparametric Kruskal-Wallis test with Dunn's post-test was used to compare differences among multiple groups.

\section{RESULTS}

\section{Generation of $L$. (L.) Amazonensis Expressing the tdTomato Protein}

The pIR1SAT-tdTomato plasmid was linearized with SwaI restriction enzyme before transfection of $L$. (L.) amazonensis parasites (Figure 1A). The linearized plasmid integrates into the Leishmania genome by replacing one copy of the SSU rRNA gene which is transcribed by pol1 (Buckner and Wilson, 2005). After the selection of transfected parasites with NTC, 6 clones out of the 23 (C2, C3, C5, C11, C12 and C21) were chosen randomly to confirm the genomic integration by PCR. The Figure 1B shows a schematic representation of the expected genomic loci after transfection and the primers used for PCR of selected clones. The pair of primers 3001-F and 3002-R hybridized outside the expression cassette and were used to corroborate insertion of the linearized plasmid. As we expected, PCR fragments of $1.1 \mathrm{~kb}$ (Figure 1C) and 2.1-2.9 kb (Figure 1D) were obtained when primers sets 3001-F/TOMA-R and F2999/3002-R were used respectively in the analysis of the 6 clones selected.

\section{Detection of Fluorescence Signal in Transfected Parasites and Its Correlation With the Number of Parasites}

The fluorescence signal was detected in the six clone selected (C2, $\mathrm{C} 3, \mathrm{C} 5, \mathrm{C} 11, \mathrm{C} 12$ and $\mathrm{C} 21$ ) after $24 \mathrm{~h}$ of incubation at $23^{\circ} \mathrm{C}$ (Figure 1E). Clone 11 was the only one that showed a lower fluorescence with respect to the others $(p<0.05)$ (Figure 1E); this could be related with the kinetic of different clone cultures, i.e. parasites in different stages of growth could be slightly different in its metabolism, affecting the transcriptional activity of the reporter gene.
A

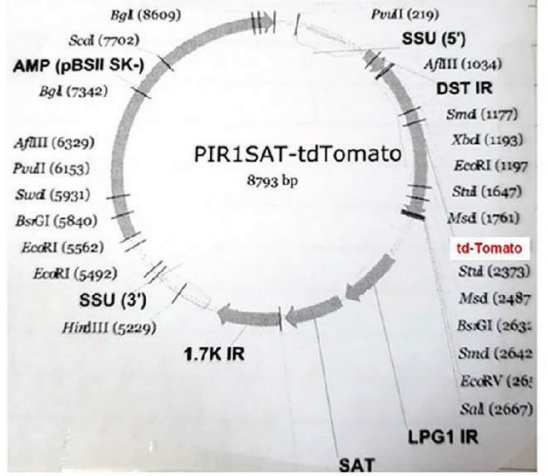

E

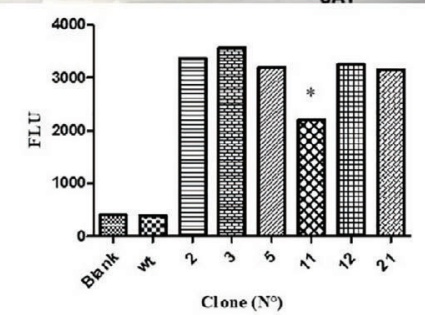

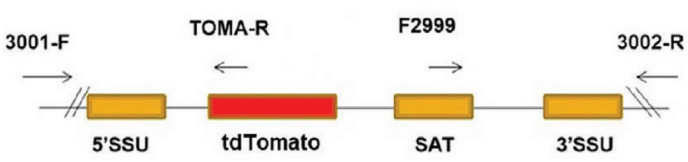

C
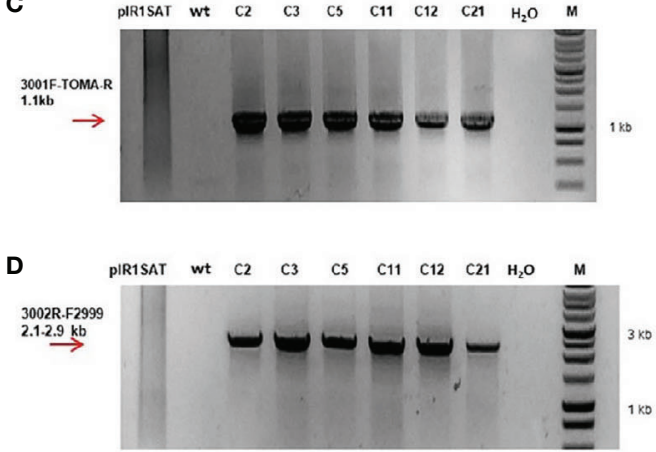

FIGURE 1 | (A) Map of the PIR1SAT-tdTomato plasmid indicating the enzyme used for cloning and linearization for $L$. (L.) amazonensis transfection assays. (B) Schematic representation of expected genomic loci of transfected Leishmania after replacement of one copy of the SSU rRNA gene. (C) PCR genotyping analysis with primers 3001-F/TOMA-R confirming the expected gene insertion. (D) PCR genotyping analysis with primers 3002-R/F2999 also corroborating the correct insertion and replacement of SSU rRNA gene. Line 1: pIR1SAT-tdTomato plasmid, line 2: wild type parasites, lines 3-8: transfected clones, line 9: no template control (-), line 10: molecular weight and marker. Diagrams are not to scale. Numbers are sizes (kb) of expected products. (E) Detection of fluorescence emission in six clone selected (C2, C3, C5, C11, C12 and C21) after $24 \mathrm{~h}$ of incubation at $23^{\circ} \mathrm{C}\left({ }^{*} p<0.05\right)$. Parasite wild-type (wt). Statistical significance was assessed using ANOVA $\left({ }^{*} p<0.05\right)$. 
The clone number 3 (L.amaz/tc3) was selected to carried out the subsequent in vitro and in vivo experiments. Promastigotes and amastigotes of $L$. amaz/tc3 showed a good correlation between the fluorescence signal and the number of parasites $(p<0.0001)$ (Figures 2A, B).

\section{In Vitro Assessment of Transfected Parasites Growth}

On the first day of culture, the density of both, L. amaz/tc3 and L. amaz/wt, increased almost 20 times (approximately to $1 \times 10^{7}$ $\mathrm{p} / \mathrm{ml}$ ). Then, from day 3 to 7 , the density of fluorescent parasites and wild-type was stable showing the cultures were in the stationary phase (Figure 2C). No statistical differences were observed between the curve of growth of $L$. amaz/tc3 and L. amaz/wt, indicating that transfection with the RG did not modified the normal parasite growth.

In addition, the growth of intracellular amastigotes of L. amaz/tc3 was evaluated at different times and ratios of infection. The fluorescence signal increased with the infection time and reached a peak on the second day post-infection (pi) (Figure 2D). Besides, the fluorescence was significantly higher in macrophages infected at ratio 1:20 with respect to those infected at radio 1:5 $(p<0.001)$ and 1:10 $(p<0.05)$ (Figure 2D). After that, the fluorescence started to decrease in all the infected cultures $\left(4^{\text {th }}\right.$ day pi, data not shown).

\section{Validation of the Fluorescence Assay}

Once the fluorescence was detected in promastigote and amastigote of $L$. amaz/tc3, we evaluated if the fluorescence was a good indicator of the parasite viability after treatment with an anti-leishmanial drug. For that, MI was selected since it showed activity against promastigotes and amastigotes of several species of Leishmania, and the efficacy of MI was determined by the fluorescent assay and by the conventional methods. After, $48 \mathrm{~h}$ of incubation with $\mathrm{MI}$, promastigotes viability decrease, and no difference was observed between the values of $\mathrm{IC}_{50}$ determined by the fluorescence and microscopic test $\left(\mathrm{IC}_{50}=5.25 \pm 0.05 \mu \mathrm{g} /\right.$ $\mathrm{ml})$. In the same manner, $\mathrm{MI}$ inhibited the growth of intracellular amastigotes, and both methods showed similar results $\left(\mathrm{IC}_{50}=\right.$ $1.65 \pm 0.75 \mu \mathrm{g} / \mathrm{ml})$.

\section{Course of Infection of $L$. amaz/tc3 in Mice}

The infectivity of $L$. amaz/tc3 was evaluated in BALB/c mice. After infection, the footpad swelling increased weekly as did the wild strain. At week 5 pi, no differences in the footpad swelling were observed (Figure 3A).

\section{Efficacy of Ma in Mice Infected With L. amaz/tc3}

$\mathrm{BALB} / \mathrm{c}$ mice were infected with $L$. amaz/tc3 to evaluate the efficacy of Ma. The animals were treated with the antimonial for 21 days, once a day. After one week of treatment, the footpad swelling started to decrease and it still continued after ended the treatment (week 12-13) (Figure 3B). At week 13 of the experiment, a significant difference in the footpad swelling was observed between $\mathrm{Ma}$ and the control group $(p<0.05)$ (Figure 3B). In addition, differences highly significant were observed in the parasite burden between $\mathrm{Ma}$ and control group $(p<0.001)$ for FL and OM showing 55\% and 75\% of inhibition, respectively. On the other hand, we observed the parasite burden determined by LD assay was significantly lower $(p<0.001)$ in both groups, $\mathrm{Ma}$ and control, than those quantified by FL and $\mathrm{OM}$; despite this, the inhibition percentage obtained by this assay was 54\%, similar to those obtained for FL (Figures 3C, D).
A

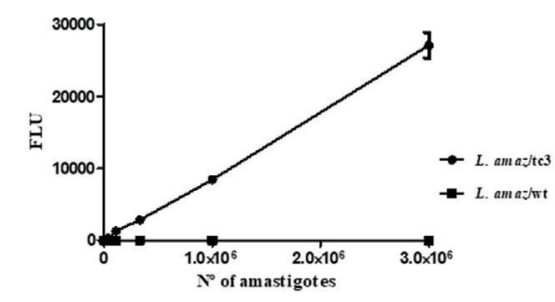

C

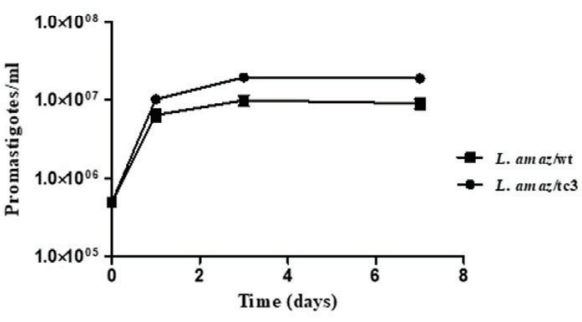

B

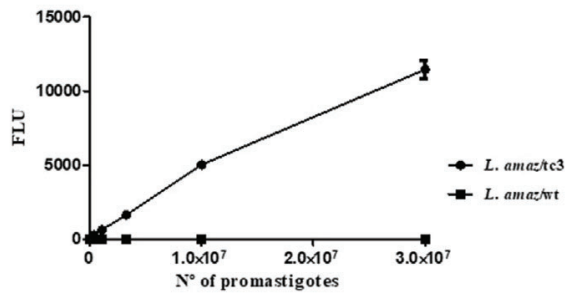

D

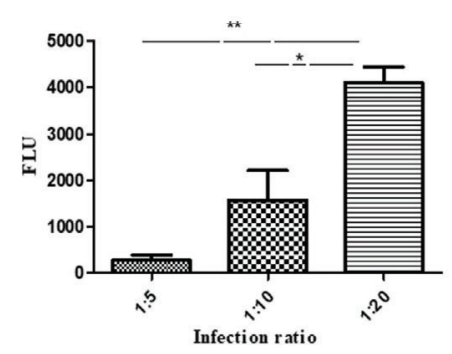

FIGURE 2 | Correlation between the fluorescence signal and the number of amastigotes (A) and promastigotes of $L$. amaz/tc3 (B). (C) No statistical differences were observed between the curve of growth of $L$. amaz/tc3 and L. amaz/wt, indicating that transfection with the RG did not modified the normal parasite growth. (D) The fluorescence signal of intracellular amastigotes of $L$. amaz/tc3 increased reaching the peak at the second day post-infection, and it was higher in macrophages infected with the ratio 1:20 than 1:5 and 1:10. Statistical significance was assessed using ANOVA $\left({ }^{*} p<0.05\right.$ and $\left.{ }^{* \star} p<0.001\right)$. 


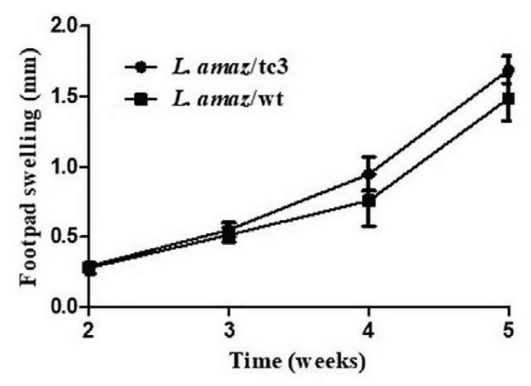

C

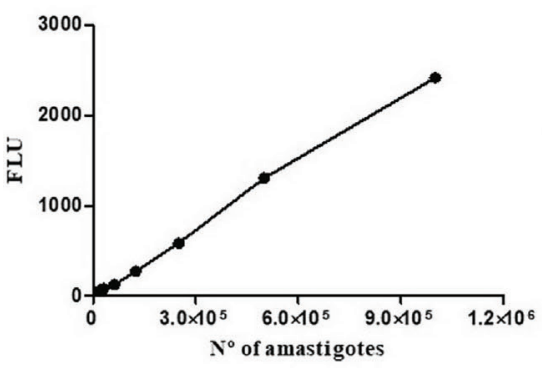

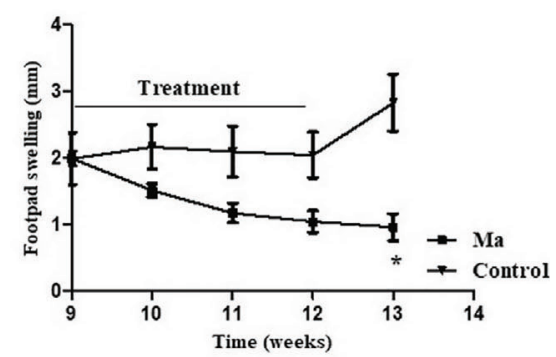

D

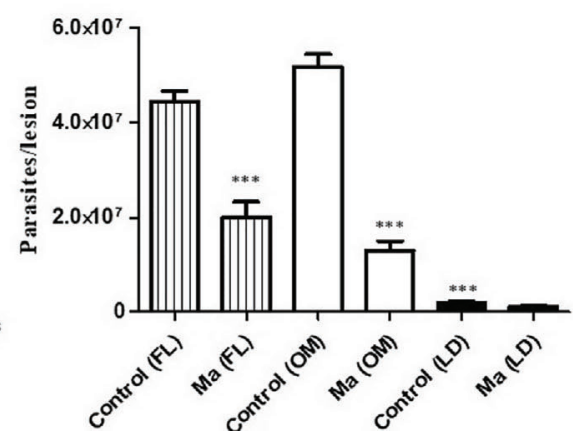

FIGURE 3 | (A) The infectivity of $L$. amaz/tc3 in BALB/c mice was similar to the wild strain (L. amaz/wt). (B) Reduction in the footpad swelling in BALB/c mice infected with $L$. amaz/tc3 and treated with meglumine antimoniate (MA) $(120 \mathrm{mg} \mathrm{Sb} / \mathrm{kg} / \mathrm{day})(p<0.05)$. (C) Calibration curve, fluorescence and number of amastigotes isolated from mice infected with $L$. amaz/tc3 $\left(r^{2}=0.99, p<0.0001\right)$. (D) Parasite load quantified, at week 13 of the experiment in mice treated with Ma and control group, by fluorescence method (FL), optic microscope (OM) and limiting dilution (LD). Statistical significance was assessed using Mann-Whitney U-test $t$ $\left({ }^{\star} p<0.05\right)$ and ANOVA $\left({ }^{\star \star \star} p<0.001\right)$. Data (mean \pm SD) are from a representative experiment $(n=5$ mice).

\section{Drug Screening Against Promastigotes and Intracellular Amastigotes by the Fluorescence Assay}

First, the anti-leishmanial activity of a group of flavonols (GAL, $\mathrm{MO}, \mathrm{FI}$ and RU) was assessed in promastigotes cultures. Also, we compared the activity of this group with EGCG, a catechin flavanol with leishmanicidal activity that we reported previously (Sosa et al., 2020). GAL showed activity on L. amaz/tc3 and the $\mathrm{IC}_{50}$ value was 2.2 fold lower $(53.09 \pm 16.56 \mu \mathrm{M})$ than EGCG $(119.8 \pm 17.6 \mu \mathrm{M})$; while no inhibition in the parasite growth was observed for MO, FI and RU with doses $\leq 40 \mu \mathrm{M}$. The reference drug $\mathrm{PE}$, used as the positive control, was highly active against promastigotes $\left(\mathrm{IC}_{50}=0.67 \pm 0.18 \mu \mathrm{M}\right)$ after $48 \mathrm{~h}$ of incubation.

Then, we evaluated the efficacy of flavonols (GAL, MO, FI and RU), EGCG and the reference drug (Ma) in the macrophage-amastigote model by the fluorescent assay. After treatment, the fluorescence was measured in the plate reader. The results caught our attention, since no relationship between the drug concentrations and the fluorescence was observed in GAL, $\mathrm{MO}$ and FI. The background fluorescence increased with concentrations $\geq 30 \mu \mathrm{M}$ (GAL and FI) or $\geq 75 \mu \mathrm{M}$ (MO), and it could neither be subtracted from the blank nor be eliminated by washing the cell culture. It is important to mention, the autofluorescence of macrophage cells and culture medium was very low compared to the fluorescence of td-tomato (data not shown). Therefore, we could not determine the $\mathrm{IC}_{50}$ for GAL, $\mathrm{MO}$ and FI by the fluorescent assay; instead, we estimated the percentage of amastigotes inhibition in those concentrations where the background did not interfere the fluorescence readout. GAL and FI showed a similar percentage of inhibition on the parasites (29-31\%) with $15 \mu \mathrm{M}$ (Table 1). On the other hand,

TABLE 1 | In vitro anti-leishmanial activity and cytotoxicity of flavonoids and the reference drug, MA.

\begin{tabular}{|c|c|c|c|c|c|c|c|}
\hline METHOD & & GAL & MO & $\mathbf{R U}$ & $\mathbf{F I}$ & EGCG & Ma \\
\hline Microscopic & $\mathrm{IC}_{50} \mu \mathrm{M}$ (SE) & $20.59( \pm 4.47)$ & $122.4( \pm 2.55)$ & $133( \pm 8.25)$ & $>60$ & $130( \pm 10.01)$ & ND \\
\hline \multirow[t]{2}{*}{ Fluorescence } & $\mathrm{IC}_{50} \mu \mathrm{M}$ (SE) & ND & ND & 171 & ND & ND & $80.31( \pm 14.7)$ \\
\hline & Inhibition (\%) & 29 & 13.5 & ND & 31 & 20 & ND \\
\hline \multirow[t]{2}{*}{ Colorimetric } & $\mathrm{CC}_{50} \mu \mathrm{M}$ (SE) & $22.75( \pm 2.87)$ & $>230.2$ & $>240$ & $52.57( \pm 6.14)$ & $193.9( \pm 23.8)$ & $>1,000$ \\
\hline & SI & 1.1 & $>1.8$ & $>1.8$ & $<1$ & 1.5 & $>12.5$ \\
\hline
\end{tabular}

ND, Not determined; SE, Standard error; SI, Selectivity index $=C_{50}$ for macrophages $/ I C_{50}$ for Leishmania. 
MO showed a low inhibition (13.5\%) against amastigotes at $37.5 \mu \mathrm{M}$. In the case of RU and $\mathrm{Ma}$, not background fluorescence was detected, and the $\mathrm{IC}_{50}$ values were $171 \mu \mathrm{M}$ and $80.31 \mu \mathrm{M}$, respectively (Table $\mathbf{1}$ ).

Then, to estimate the $\mathrm{IC}_{50}$ values of GAL, FI and MO; and to confirm the $\mathrm{IC}_{50}$ of $\mathrm{RU}$, the activity was evaluated quantifying the intracellular amastigotes at the optic microscope. We followed the same methodology described for MI (microscopic assay) in "In Vitro, Fluorescent Assay Validation" section and the range of concentrations were for GAL (30 to $7.5 \mu \mathrm{M}$ ), FI (60 to 15 $\mu \mathrm{M}), \mathrm{RU}$ and $\mathrm{MO}$ (200 to $50 \mu \mathrm{M})$, EGCG (142 a $17.7 \mu \mathrm{M})$. The $\mathrm{IC}_{50}$ of GAL $(20.59 \pm 4.47 \mu \mathrm{M})$ was 5.9 and 6.4 times lower than the $\mathrm{IC}_{50}$ of $\mathrm{MO}(122.4 \pm 2.55 \mu \mathrm{M})$ and $\mathrm{RU}(133 \pm 8.25 \mu \mathrm{M})$, respectively. Furthermore, GAL was more active than the flavanol EGCG $\left(\mathrm{IC}_{50}=130 \pm 10.01 \mu \mathrm{M}\right)$ (Table 1).

Referred to the toxicity, GAL (SI = 1.1), MO (SI > 1.8), RU $(\mathrm{SI}>1.8)$, EGCG $(\mathrm{SI}=1.5)$ and $\mathrm{Ma}(\mathrm{SI}>12.5)$ were more selective against the parasites than the host cells with SI values higher than 1. Instead, FI was more selective against the host cells than the parasite $(\mathrm{SI}<1)$ (Table 1$)$.

\section{GAL and EGCG Induced Ultrastructural Alterations in Leishmania}

Through the in vitro drug screening, we found GAL was the most active compound against Leishmania. Thus, we investigated the effects of this compound on the ultrastructure of L. amaz/tc3 promastigotes by transmission electron microscopy (TEM). In addition, we analyzed the effects of EGCG, another compound that also showed anti-leishmanial activity. As controls we used untreated L. amaz/tc3 promastigotes. As we expected, control promastigotes presented a typical elongated morphology with normal cellular structures such as nucleus, lipid inclusion, mitochondrion, and flagellar pocket (Figure 4A). Both GAL and EGCG induced different alterations in the ultrastructure of promastigotes, showing intense cellular damage (Figures 4B, C). GAL produced dramatic changes in the mitochondrial structure, with the formation of enlarged and swollen mitochondria compared to the untreated control, cytoplasmic lipid bodies as well an increase in plasma membrane blebs (surface blebbing) (Figure 4C). EGCG induced intense cytoplasmic vacuolization and also the presence of nuclear membrane detachment, chromatin condensation, and marginalization (Figure 4B). Moreover, the morphology of the parasites exposed to the drugs became visibly altered, with loss of most of the cytoplasmic organelles and a predominant round shape, confirming cellular death.

\section{GAL Induced the Production of Th1 Cytokine in Infected Cultured Macrophages}

We determined in the culture supernatant the concentration of two cytokines produced by activated macrophages: IL-12 p70, required for the induction of IFN- $\gamma$ production; and IL- 10 , which is known to diminish the production of inflammatory mediators. We compared the production of IL-12 p70 and IL-10 between the different culture treated cells: infected and non-infected macrophages alone (RPMI medium) or exposed to PHA, CONA, and GAL. GAL was evaluated in this assay since this compound was the most active against Leishmania. Infected cells treated with GAL showed higher production of IL-12 p70 [mean (range) $232.2 \mathrm{pg} / \mathrm{ml}(137.5-331.7 \mathrm{pg} / \mathrm{ml})$ ] compared to infected non-stimulated controls (RPMI medium) $[72.11 \mathrm{pg} / \mathrm{ml}$ (56.38$84.20 \mathrm{pg} / \mathrm{ml})$ ] $(p<0.01)$ (Figure 5A). Moreover, the addition of GAL to infected macrophages induced a significant increase of IL-12 p70 in comparison to non-infected macrophages treated with the same stimulus $(p<0.05)$ (Figure 5B). IL-10 concentration did not show significant differences between the different treated groups (data not shown).

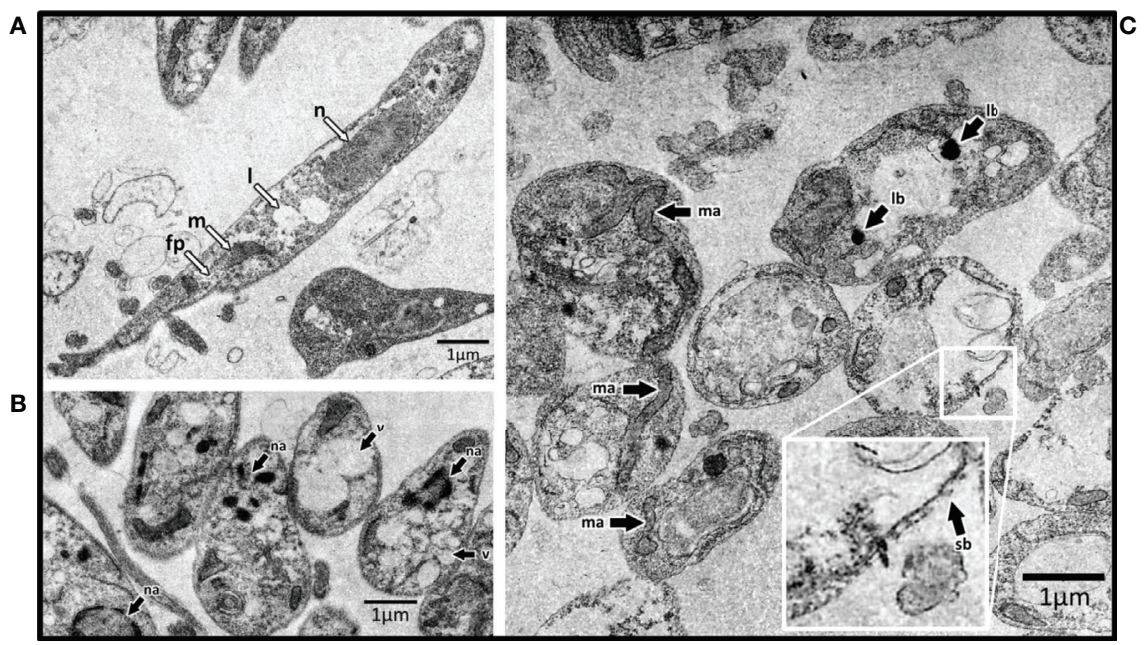

FIGURE 4 | Transmission electron microscopy of L. amaz/tc3 promastigotes. (A) Untreated culture with normal cellular structures: nucleus (n), lipid inclusion (I), mitochondrion (m), and flagellar pocket (fp). Black arrows indicate ultrastructural alterations in parasites exposed to EGCG (B) and GAL (C) nuclear alterations (na), vacuolization (v), alterations in mitochondrial structure (ma), lipid-storage bodies (lb), and surface blebbing (sb). 
A

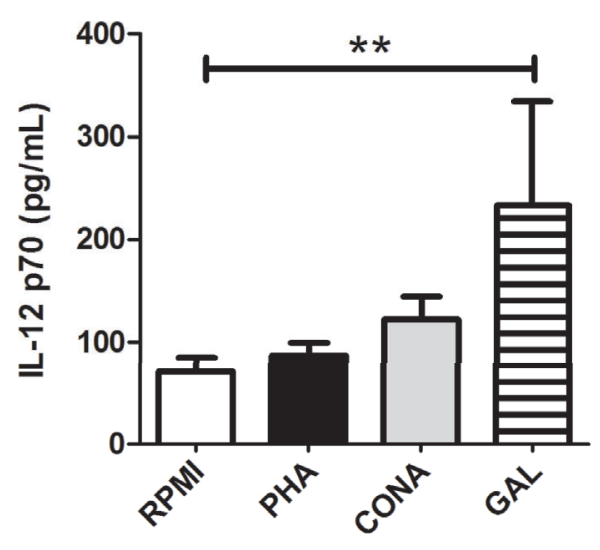

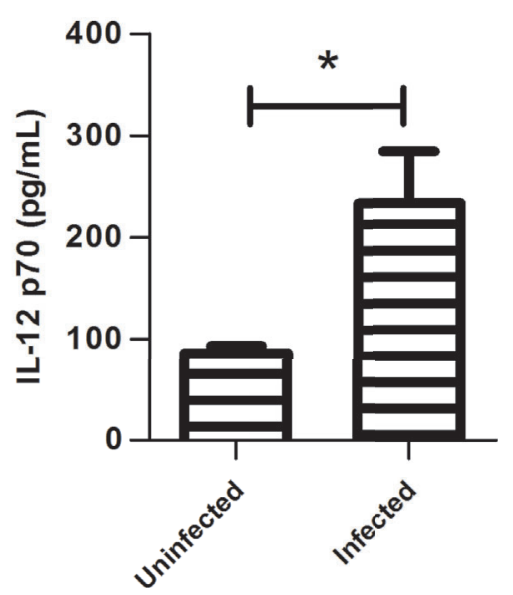

FIGURE 5 | Culture supernatant production of IL-12 p70 by Leishmania infected macrophages treated with GAL. (A) The amount of IL-12p70 was assessed in infected cultured RAW 264.7 macrophages maintained alone (RPMI) or treated with phytohemagglutinin (PHA), concanavalin A (CONA) and galangin (GAL). Each variable was performed in quadruplicate. Comparison among groups were assessed by Kruskal-Wallis test and Dunn`s post-test $\left({ }^{* *} p<0.01\right)$. (B) Production of IL12 p70 after the addition of GAL to uninfected vs. infected cultured macrophages. Analysis performed by Mann Whitney test $\left({ }^{*} p<0.05\right)$.

\section{DISCUSSION}

A search for new chemotherapeutic compounds for leishmaniasis diseases is still a challenge as well as the development of new methods to evaluate potential anti-leishmanial drugs. Moreover, the emergence of drug resistance and the treatment failure to antimonial make this search imperative (Agnew et al., 2001; García-Bustos et al., 2021).

In this sense, we obtained a clone of $L$. (L.) amazonensis ( $L$. amaz/tc3) expressing the tdTomato. Its tandem dimer structure plays an important role in the exceptional brightness of this protein (283\% of eGFP and $160 \%$ of DsRed) (Shaner et al., 2004). It is important to mention, that tdTomato gene was integrated into Leishmania genome by replacing a copy of SSU rRNA gene; this region has a high expression level since it is transcribed by polymerase 1, and it is highly conserved among Leishmania species (Buckner and Wilson, 2005).

As was expected, promastigotes and amastigotes of L. amaz/ tc3 showed a strong fluorescence signal, and it was correlated with the number of parasites. On the other hand, the genetic manipulation of parasites did not alter either, the in vitro growth, or the infectivity in BALB/c mice showing similar behavior to the wild-type strain (L.amaz/wt).

In vitro, the fluorescence assay was validated with MI since promastigote are not sensitive to MA (Robert et al., 1995). MI showed a similar efficacy against both $L$. amaz/tc3 and the wild type (L. amaz/wt), indicating that transfection and the constitutive tdTomato expression did not affect the anti-leishmanial activity of the drug. The $\mathrm{IC}_{50}$ values determined for MI by the fluorescence assay in our study was consistent with the published data that demonstrated an effective dose of $1.31 \mu \mathrm{g} / \mathrm{ml}$ and $7.54 \mu \mathrm{g} / \mathrm{ml}$ for amastigotes and promastigotes of $L$. (L.) amazonensis, respectively (Escobar et al., 2002; Morais-Teixeira et al., 2011).
Then, the fluorescence assay was validated in vivo. BALB/c mice were infected with L. amaz/tc3 and L. amaz/wt and treated with Ma, the drug efficacy was determined by fluorescence and LD assay. Both methods showed a similar percentage of inhibition in the parasite load (54-55\%) at the end of treatment, but the fluorescence assay was more sensitivity than the LD. The sensitivity difference observed between the methods might be because LD assay only detect amastigotes capable of transforming into promastigotes and surviving in the medium culture; instead, the fluorescence assay quantifies all the amastigotes present in the crude extract of lesion macerate able of expressing the tdTomato protein. Besides, the parasite load determined by the fluorescent assay was consistent with the optic microscope indicating the amastigotes were viable. In agreement with our results, the luminescence assay in ex vivo tissue also showed a higher sensitivity than the LD (Reimão et al., 2013).

The fluorescence assay developed in this work, through the expression of tdTomato RG, was a simple system for drug screening. This method, unlike those based on catalytic reporter genes ( $\beta$-lactamase, luciferase, $\beta$-galactosidase) (Okuno et al., 2003; Ashutosh et al., 2005; Buckner and Wilson, 2005), has the advantage that not require an addition of reagent, and the fluorescence readout can be done at different times of incubation. Besides, we highlight the stability of the fluorescence of $L$. amaz/tc3. We observed that transfected parasites showed fluorescence 6 months' post-infection in $\mathrm{BALB} / \mathrm{c}$ mice (data not shown). The fluorescence stability in infected animals is important in studies that require a long period of evaluation like relapses of treatments or in vaccines studies. Furthermore, these transgenic parasites can be used at in-vivo experiments taking advantage of the tdTomato emission range, which is easily detected at $620 \mathrm{~nm}$, outside of the range of most of the animal tissue auto-fluorescence (Canavaci et al., 2010). 
Several fluorescent proteins, applied in drug screening assay, have been studied during the last three decades. The GFP was the most studied, but the main disadvantage of this RG was the low level of fluorescence of transgenic parasites (Ha et al., 1996). Then, trying to improve the fluorescent signals, egfp was studied. The transfected parasites showed stable expression of the fluorescent protein and the assay provided a more accurate approach in drug sensitivity profile, but it was not automated as a high-throughput (Bolhassani et al., 2011). On the contrary, our system was automatized using a plate reader, to measure the fluorescence in promastigotes drug screening and in amastigotes isolated from treated animals.

Through the fluorescence assay, we selected the flavonol GAL as the most active compound against promastigotes of L. amaz/ tc3. Another work reported the activity of GAL isolated from Alpinia galanga rhizomes against promastigotes of $L$. (L.) donovani, the causal agent of visceral leishmaniasis, but the parasite inhibition was reached at $100 \mu \mathrm{M}\left(\mathrm{IC}_{50}\right)$, (Kaur et al., 2010) almost twice the $\mathrm{IC}_{50}$ in $L$. amaz/tc3 determined in this work. The differences in the sensitivity might be related to the species of Leishmania as it was showed by Escobar et al. (2002).

It is worth to mention that in the macrophage-amastigote model, we observed an interference in the tdTomato fluorescence readout, and it increased with drug concentrations of GAL, MO and FI. This interference was not associated with the autofluorescence of macrophages and culture medium because they were very low compared to the tdTomato. Mukai et al. (2009) demonstrated that flavonol aglycones like GAL, MO, isorhamnetin and quercetin, presented auto-fluorescence (ex $488 \mathrm{~nm}$ and em 515-535 nm) in Hepa-1c1c7 cells treated with these compounds; moreover, it was concentration-dependent. They suggested the auto-fluorescence might be related with the hydroxyl group at the C3-position in the flavonol skeleton since no fluorescence was observed in the cells treated with compounds belonging to the flavone, flavanone and catechin subclasses. Therefore, we hypothesized the interference in the tdTomato readout might be due to an overlap between the extremes of the emission spectra of tdTomato and green fluorescence of the flavonol aglycones when they are excited at $535 \mathrm{~nm}$. On the other hand, we did not observe interference in the tdTomato fluorescence readout when infected macrophages were treated with RU and $\mathrm{Ma}$ and we could determine the $\mathrm{IC}_{50}$ of these compound. The activity of GAL, MO, FI and EGCG was determined by optic microscopy.

After all, GAL was the most active compound against promastigotes and amastigotes of $L$. amaz/tc3. GAL produced morphological alterations on mitochondria and plasma membrane of promastigotes of $L$. amaz/tc3 with loss of the cytoplasmic organelles. Moreover, the ability of GAL to induce IL-12p70 up-regulation by Leishmania infected macrophages in vitro indicates that this natural compound could play an important role in the eradication of the invading parasites. Optimal production of both fractions of IL-12 (p40 and p70) is required to drive a predominant $\mathrm{T}$ helper 1 response with the subsequent IFN- $\gamma$ production, macrophage activation and NOmediating effects needed to kill the pathogens (Mattner et al., 1996; Guler et al., 2011).
All these data demonstrates that the fluorescence assay, based on the stable expression of the tdTomato protein by $L$. (L.) amazonensis, is a useful tool to assess the anti-leishmanial activity of new drugs. However, some interference in the tdTomato readout can occur in the macrophage-amastigote model when flavonol aglycones are been testing. Further studies are necessary to identify if there are others natural compounds that might affect the performance of the fluorescence assay. Besides, the present study demonstrated the potential inhibitory effect of GAL against $L$. (L.) amazonensis and its capacity to induce the production of IL-12 p70, a cytokine related to a Th1 response (cure); therefore, these results encourage us to study its efficacy in animal model in near future.

\section{DATA AVAILABILITY STATEMENT}

The original contributions presented in the study are included in the article/Supplementary Material. Further inquiries can be directed to the corresponding author.

\section{ETHICS STATEMENT}

The animal study was reviewed and approved by Animal Ethics Committee of the Faculty of Health Sciences, National University of Salta, Salta, Argentina (Resolution N ${ }^{\circ} 309-18$ ).

\section{AUTHOR CONTRIBUTIONS}

MG-B, PB, CPB, AM, CP, JM, AS, VB, OP, PM, and PP performed experiments. MG-B, $\mathrm{PB}, \mathrm{CPB}, \mathrm{CP}$, and JM analyzed data. MG-B and $P B$ designed experiments. $M G-B$ and $P B$ drafted the manuscript. All authors contributed to the article and approved the submitted version.

\section{FUNDING}

This work was funded by Agencia Nacional de Promoción Científica y Tecnológica and by GLAXO, PICT-O 2011-0017 (PB), by the Research Council of the Catholic University of Salta, Argentina (Grant Resolution 1494/16) and by the Research Council of the National University of Salta (Type B Project No. 2411) (MG-B).

\section{ACKNOWLEDGMENTS}

The authors thank to Med. Vet. Maria Celia Mora and to the technicians Alejandro Uncos, Federico Ramos and Renato Uncos for the technical supports in vivo assays; and to the technicians Cecilia Gallardo, Walter Ferrari and Manuel Siñeriz Louis for technical assistance in the TEM experiments. We also thank to Dr. Carl Taswell who kindly provided us the ELIDA software, to 
Dr. Stephen Beverley for providing us the pIR1SAT plasmid, and to Dr. R.L. Tarleton who provide us the pTREX-tdTomato plasmid. Finally, we thank to Mitsui Norin (Shizuoka, Japan) for providing us the EGCG, and to the Ministerio de Salud de la Nación (Buenos Aires, Argentina) for supplying us the MA.

\section{REFERENCES}

Adinehbeigi, K., Razi Jalali, M. H., Shahriari, A., and Bahrami, S. (2017). In Vitro Antileishmanial Activity of Fisetin Flavonoid Via Inhibition of Glutathione Biosynthesis and Arginase Activity in Leishmania Infantum. Pathog. Glob. Health 111, 176-185. doi: 10.1080/20477724.2017.1312777

Agnew, P., Holzmuller, P., Michalakis, Y., Sereno, D., Lemesre, J. L., and Renaund, F. (2001). In Vitro Growth of Leishmania Amazonensis Promastigotes Resistant to Pentamidine Is Dependent on Interactions Among Strains. Antimicrob. Agents Chemother. 45, 1928-1929. doi: 10.1128/AAC.45.6.1928-1929.2001

Alvar, J., Vélez, I. D., Bern, C., Herrero, M., Desjeux, P., Cano, J., et al. (2012). Leishmaniasis Worldwide and Global Estimates of its Incidence. PloS One 7, e35671. doi: 10.1371/journal.pone.0035671

Ashutosh,, Gupta, S., Ramesh,, Sundar, S., and Goyal, N. (2005). Use of Leishmania Donovani Field Isolates Expressing the Luciferase Reporter Gene in In Vitro Drug Screening. Antimicrob. Agents Chemother. 49 (9), 3776-3783. doi: 10.1128/AAC.49.9.3776-3783.2005

Ayres, D. C., Pinto, L. A., and Giorgio, S. (2008). Efficacy of Pentavalent Antimony, Amphotericin B, and Miltefosine in Leishmania AmazonensisInfected Macrophages Under Normoxic and Hypoxic Conditions. J. Parasitol. 94, 1415-1417. doi: 10.1645/GE-1613.1

Bolhassani, A., Taheri, T., Taslimi, Y., Zamanilui, S., Zahedifard, F., Seyed, N., et al. (2011). Fluorescent Leishmania Species: Development of Stable GFP Expression and its Application for In Vitro and In Vivo Studies. Exp. Parasitol. 127 (3), 637-645. doi: 10.1016/j.exppara.2010.12.006

Buckner, F. S., and Wilson, A. J. (2005). Colorimetric Assay for Screening Compounds Against Leishmania Amastigotes Grown in Macrophages. Am. J. Trop. Med. Hyg. 72, 600-605. doi: 10.4269/ajtmh.2005.72.600

Canavaci, A. M. C., Bustamante, J. M., Padilla, A. M., Perez Brandan, C. M., Simpson, L. J., Xu, D., et al. (2010). In Vitro and In Vivo High-Throughput Assays for the Testing of Anti-Trypanosoma Cruzi Compounds. PloS Negl. Trop. Dis. 4, e740. doi: 10.1371/journal.pntd.0000740

Chang, K. P. (1980). Human Cutaneous Leishmania in a Mouse Macrophage Line: Propagation and Isolation of Intracellular Parasites. Science 209, 1240-1242. doi: $10.1126 /$ science. 7403880

Chouhan, G., Islamuddin, M., Sahal, D., and Afrin, F. (2014). Exploring the Role of Medicinal Plant-Based Immunomodulators for Effective Therapy of Leishmaniasis. Front. Immunol. 5, 193. doi: 10.3389/fimmu.2014.00193

Dube, A., Gupta, R., and Singh, N. (2009). Reporter Genes Facilitating Discovery of Drugs Targeting Protozoan Parasites. Trends Parasitol. 25, 432-439. doi: 10.1016/j.pt.2009.06.006

Dube, A., Singh, N., Sundar, S., and Singh, N. (2005). Refractoriness to the Treatment of Sodium Stibogluconate in Indian Kala-Azar Field Isolates Persist in In Vitro and In Vivo Experimental Models. Parasitol. Res. 96, 216-223. doi: 10.1007/s00436-005-1339-1

Escobar, P., Matu, S., Marques, C., and Croft, S. L. (2002). Sensitivitites of Leishmania Species to Hexadecylphosphocholine (Miltefosine), ET-18OCH3 (Edelfosine) and Amphotericin B. Acta Trop. 81, 151-157. doi: 10.1016/S0001-706X(01)00197-8

García-Bustos, M. F., González-Prieto, G., Pániz-Mondolfi, A. E., Parodi, C., Beckar, J., Monroig, S., et al. (2021). Risk Factors for Antimony Treatment Failure in American Cutaneous Leishmaniasis in Northwestern-Argentina. PloS Negl. Trop. Dis. 15, e0009003. doi: 10.1371/journal.pntd.0009003

Guler, R., Afshar, M., Arendse, B., Parihar, S. P., Revaz-Breton, M., Leitges, M., et al. (2011). PKC $\delta$ Regulates IL-12p40/p70 Production by Macrophages and Dendritic Cells, Driving a Type 1 Healer Phenotype in Cutaneous Leishmaniasis. Eur. J. Immunol. 41, 706-715. doi: 10.1002/eji.201040985

Ha, D. S., Schwarz, J. K., Turco, S. J., and Beverley, S. M. (1996). Use of the Green Fluorescent Protein as a Marker in Transfected Leishmania. Mol. Biochem. Parasitol. 77, 57-64. doi: 10.1016/0166-6851(96)02580-7

\section{SUPPLEMENTARY MATERIAL}

The Supplementary Material for this article can be found online at: https://www.frontiersin.org/articles/10.3389/fcimb. 2021.666746/full\#supplementary-material

Hill, J. O., North, R. J., and Collins, F. M. (1983). Advantages of Measuring 833 Changes in the Number of Viable Parasites in Murine Models of Experimental Cutaneous Leishmaniasis. Infect. Immun. 39, 1087-1094. doi: 10.1128/ IAI.39.3.1087-1094.1983

Kaur, A., Singh, R., Dey, C. S., Sharma, S. S., Bhutani, K. K., and Singh, I. P. (2010). Antileishmanial Phenylpropanoids From Alpinia Galanga (Linn.) Willd. Indian J. Exp. Biol. 48, 314-317.

Lima, H. C., Bleyenberg, J. A., and Titus, R. G. (1997). A Simple Method for Quantifying Leishmania in Tissues of Infected Animals. Parasitol. Today 13, 80-82. doi: 10.1016/S0169-4758(96)40010-2

Manjolin, L. C., dos Reis, M. B., Maquiaveli do Carmo, C., Santos-Filho, O. A., and da Silva, E. R. (2013). Dietary Flavonoids Fisetin, Luteolin and Their Derived Compounds Inhibit Arginase, A Central Enzyme in Leishmania (Leishmania) Amazonensis Infection. Food. Chem. 141, 2253-2262. doi: 10.1016/j.foodchem.2013.05.025

Mattner, F., Magram, J., Ferrante, J., Launois, P., Di Padova, K., Behin, R., et al. (1996). Genetically Resistant Mice Lacking Interleukin-12 Aresusceptible to Infection With Leishmania Major and Mount a Polarized Th2cell Response. Eur. J. Immunol. 26, 1553-1559. doi: 10.1002/eji.1830260722

Mcgwire, B. S., and Satoskar, A. R. (2014). Leishmaniasis: Clinical Syndromes and Treatment. QJM 107, 7-14. doi: 10.1093/qjmed/hct116

Morais-Teixeira, E., Damasceno, Q., Galuppo, M., Kolos, R., Romanha, A. J., and Rabello, A. (2011). The In Vitro Leishmanicidal Activity of Hexadecylphosphocholine (Miltefosine) Against Four Medically Relevant Leishmania Species of Brazil. Mem. Inst. Oswaldo Cruz 106, 475-478. doi: 10.1590/S0074-02762011000400015

Morris, L. M., Klanke, C. A., Lang, S. A., Lim, F. Y., and Crombleholme, T. M. (2010). TdTomato and EGFP Identification in Histological Sections: Insight and Alternatives. Biotech. Histochem. 85, 379-387. doi: 10.3109/ 10520290903504753

Mukai, R., Shirai, Y., Saito, N., Yoshida, K., and Ashida, H. (2009). Subcellular Localization of Flavonol Aglycone in Hepatocytes Visualized by Confocal Laser Scanning Fluorescence Microscope. Cytotechnology 59, 177-182. doi: 10.1007/ s10616-009-9206-Z

New, D. C., Miller-Martini, D. M., and Wong, Y. H. (2003). Reporter Gene Assays and Their Applications to Bioassays of Natural Products. Phytother. Res. 17, 439-448. doi: 10.1002/ptr.1312

Ogeto, T. K., Odhiambo, R. A., Shivairo, R. S., Muleke, C. I., Osero, B. O., Anjili, C., et al. (2013). Antileishmanial Activity of Aloe Secundiflora Plant Extracts Against Leishmania Major. Adv. Life. Sci. Technol. 13, 9-18.

Okuno, T., Goto, Y., Matsumoto, Y., Otsuka, H., and Matsumoto, Y. (2003). Applications of Recombinant Leishmania Amazonensis Expressing Egfp or the Beta-Galactosidase Gene for Drug Screening and Histopathological Analysis. Exp. Anim. 52 (2), 109-118. doi: 10.1538/expanim.52.109

Oryan, A. (2015). Plant-Derived Compounds in Treatment of Leishmaniasis. Iran J. Vet. Res. 16, 1-19.

Pan American Health Organization (2013) Leishmaniasis in the Americas. Recommendations for Treatment. Available at: http://iris.paho.org/xmlui/ handle/123456789/7704 (Accessed December 18, 2020).

Pulido, S. A., Muñoz, D. L., Restrepo, A. M., Mesa, C., Alzate, J. F., Vélez, I. D., et al. (2012). Improvement of the Green Fluorescent Protein Reporter System in Leishmania Spp. for the In Vitro and In Vivo Screening of Antileishmanial Drugs. Acta Trop. 122 (1), 36-45. doi: 10.1016/j.actatropica.2011.11.015

Reimão, J. Q., Trinconi, C. T., Yokoyama-Yasunaka, J. K., Miguel, D. C., Kalil, S. P., and Uliana, S. R. B. (2013). Parasite Burden in Leishmania (Leishmania) Amazonensis-Infected Mice: Validation of Luciferase as a Quantitative Tool. J. Microbiol. Methods 93, 95-101. doi: 10.1016/j.mimet.2013.02.007

Robert, W. L., Berman, J. D., and Rainey, P. M. (1995). In Vitro Antileishmanial Properties of Tri- and Pentavalent Antimonial Preparations. Antimicrob. Agents Chemother. 39, 1234-1239. doi: 10.1128/aac.39.6.1234 
Shaner, N. C., Campbell, R. E., Steinbach, P. A., Giepmans, B. N. G., Palmer, A. E., and Tsien, R. Y. (2004). Improved Monomeric Red, Orange and Yellow Fluorescent Proteins Derived From Discosoma Sp. Red Fluorescent Protein. Nat. Biotechnol. 22, 1567-1572. doi: 10.1038/nbt1037

Sifaoui, I., Lopez-Arencibia, A., Martin-Navarro, C. M., Chammem, N., Reyes-Batlle, M., Mejri, M., et al. (2014). Activity of Olive Leaf Extracts Against the Promastigote Stage of Leishmania Species and Their Correlation With the Antioxidant Activity. Exp. Parasitol. 141, 106-111. doi: 10.1016/j.exppara.2014.03.002

Sosa, A. M., Moya Álvarez, A., Bracamonte, E., Korenaga, M., Marco, J. D., and Barroso, P. A. (2020). Efficacy of Topical Treatment With (-)-Epigallocatechin Gallate, a Green Tea Catechin, in Mice With Cutaneous Leishmaniasis. Molecules 25, 1741. doi: 10.3390/molecules25071741

Taswell, C. (1986). "Limiting Dilution Assays for the Separation, Characterization and Quantification of Biologically Active Particles and Their Clonal Progeny", in Cell Separation: Methods and Selected Applications. Eds. T. G. Pretlow and T. P. Pretlow (New York, NY, USA: Academic Press), 109-145.

Tiuman, T. S., Ueda-Nakamura, T., Garcia Cortez, D. A., Dias Filho, B. P., MorgadoDíaz, J. A., de Souza, W., et al. (2005). Antileishmanial Activity of Parthenolide, A Sesquiterpene Lactone Isolated From Tanacetum Parthenium. Antimicrob. Agents Chemother. 49, 176-182. doi: 10.1128/AAC.49.11.176-182.2005
Wong, I. L., Chan, K. F., Chen, Y. F., Lun, Z. R., Chan, T. H., and Chow, L. M. (2014). In Vitro and In Vivo Efficacy of Novel Flavonoid Dimers Against Cutaneous Leishmaniasis. Antimicrob. Agents Chemother. 58, 3379-3388. doi: 10.1128/AAC.02425-13

World Health Organization (2016) Leishmaniasis in High-Burden Countries: An Epidemiological Update Based on Data Reported in 2014. Available at: https:// www.who.int/wer/2016/wer9122.pdf?ua=1 (Accessed November 15, 2020).

Conflict of Interest: The authors declare that the research was conducted in the absence of any commercial or financial relationships that could be construed as a potential conflict of interest.

Copyright (C) 2021 Garcia-Bustos, Moya Álvarez, Pérez Brandan, Parodi, Sosa, Buttazzoni Zuñiga, Pastrana, Manghera, Peñalva, Marco and Barroso. This is an open-access article distributed under the terms of the Creative Commons Attribution License (CC BY). The use, distribution or reproduction in other forums is permitted, provided the original author(s) and the copyright owner(s) are credited and that the original publication in this journal is cited, in accordance with accepted academic practice. No use, distribution or reproduction is permitted which does not comply with these terms. 